\title{
Alta Fecundidad en Una COMUNIDAD NATIVA DE LA Amazonia Peruana
}

\author{
Warren M. Hern
}

Se trata de un estudio inicial de la fecundidad y los cambios culturales en una comunidad shipibo, realizado entre 1964 y 1969 que incluye los aspectos: social, ambiental, económico, cultural y sanitario. El crecimiento de la población en esta comunidad alcanza un $5 \%$ anual, cifra que es mayor a la encontrada por ot ros investigadores en otros grupos. Analiza los factores que contribuyen al crecimiento poblacional y establece, entre otras cosas, que la reproducción se inicia a una edad muy temprana y se prolonga hasta alcanzar la etapa final de la edad reproductiva. Observa que el matrimonio es universal y que su alta capacidad reproductiva se refleja en el corto espaciamiento entre un parto y otro, especialmente por disminución de la poliginia, práctica desaprobada por los misioneros.

This initial study of fertility and cultural change in a Shipibo village was carried out between 1964 and 1969 and included social, environmental, economic, cultural and health aspects. Population growth in the village is almost $5 \%$ per year, and exceeds that previously recorded for other groups. The factors that contribute to population growth are explored and, among other things, established that reproduction begins at a very early age and is prolonged to reach the final stage of reproductive age. It was found that matrimony is universal and its high reproductive capacity is reflected in the short space between births and the decrease of polygyny, a practice which is disapproved by the missionaries. 


\section{INTRODUCCION}

La tasa de incremento poblacional en América Latina, globalmente a la época del estudio actual, era $2.9 \%$, la más alta del mundo; mientras que los cálculos publicados para varios países latinoamericanos reflejan una tasa mayor a 3\% anual (Nortman, 1969; Collver, 1965). La más reciente tasa de incremento poblacional publicada para el Perú es de $2.6 \%$ (INE, 1983). El Instituto Nacional de Estadística ha revelado que más del $50 \%$ de la población peruana, tiene menos de 20 años deedad (1983). Un factor, que posiblemente tiene importancia en esta situación demográfica, es la experiencia de la gente indígena, cuyas sociedades están en estado de brusca transición cultural.

Cuando estudiaba medicina en 1964; trabajé en ẹl Hospital Amazónico "Albert Schwitzer," Yarinacocha, cerca de Pucallpa, por un período de tres meses. Más tarde visité la comunidad descrita en este artículo, con la idea de lograr una investigación etnográfica. El resultado de una encuesta general de salud de la comunidad en dicha época, señaló que el rápido incremento poblacional estaba llegando a ser una influencia importante, y tal vez una influencia predominante, sobre la vida de la comunidad. Este trabajo trata de encontrar una respuesta a tal interrogante.

\section{ANTECEDENTES}

Paococha es una comunidad indígena Shipibo situada a orillas del río Ucayali, aproximádamente $150 \mathrm{Km}$, río abajo de Pucallpa, Perú. El ambiente ecológico de la aldea corresponde al bosque tropical. La comunidad pertenece a la agrupación Shipibo-Conibo, que está distribuida unos $400 \mathrm{Km}$ a lo largo del río Ucayali y sus afluentes. Los Shipibo-Conibo son miembros de la familia lingüística Pano (Lathrap, 1970). Aunque ellos han mantenido contacto con la cultura occidental por más de 200 años, tal contacto ha sido más intenso durante las últimas décadas. Según los habitantes, Paococha se formó hace más o menos 50 años, con la colaboración de misioneros adventistas.

Los Shipibo son sedentarios, viven principalmente de la agricultura y la pesca de consumo, aunque durante los últimos veinte años han entrado a la economía de mercado cada vez más. Venden pescado seco, plátanos, y otros productos de la selva a los regatones de mestizos o en Pucallpa. Desde 1960, un número creciente de indígenas Shipibo se han trasladado a Pucallpa para 
trabajar o para vivir cerca del Hospital Amazónico "Albert Schweiter" mientras sus familiares eran tratados allí. El hospital, fundado por el Dr. Teodoro Binder en 1960, ha sido una importantefuente de atención médica y contacto intercultural para los habitantes de Paococha.

El arroz y los frijoles se han convertido en productos importantes. Antiguamente se sembraba a dos niveles en las playas durante los veranos; pero ahora con la desaparición del lago ubicado junto la comunidad ha quedado una nueva área disponible para cultivos. El lago estaba originalmente a muchos kilómetros del río, pero la erosión se llevó la barrera que había entre el río y el lago, permitiendo que el lago se vacíe durante el verano y deje un suelo rico para sembrar.

Estos cambios han sido acompañados por extensa deforestación de la selva aledaña con el propósito de sembrar otras plantas de autoconsumo y de venta en el mercado. El desbroce de la selva para la agricultura se practicaba mucho antes de que se forme la comunidad, pero todos están de acuerdo en que estas prácticas han sido mucho más intensas durante los últimos 10615 años. En 1974, una gran inundación destruyó muchas cosechas; es posible que haya sido resultado de una deforestación similar río arriba. Las inundaciones obligaron a los habitantes a trasladar sus chacras a terrenos altos a muchos kilómetros del río. Lathrap (1968) asevera que es posible mantener cultivos continuos a lo largo del Ucayali, pero se debe distinguir fronteras aluviales del río de los terrenos altos interfluviales de suelo laterítico, que son más expuestos a erosión terminal. Lathrap (1970) también señaló que los suelos fértiles a lo largo del Amazonas son bastante estrechos.

Como resultado de un contacto prolongado con el Hospital Amazónico los habitantes de Paococha están más dispuestos a buscar y aceptar atención médica occidental. Por ejemplo, las mujeres con problemas obstétricos son llevadas rápidamente al hospital municipal, $50 \mathrm{Km}$ río abajo, en Contamana. Se permite también que los funcionarios peruanos de salud pública ingresen a la comunidad para ofrecer vacunación.

La población de la comunidad dobló entre los censos de 1964 y 1969; pero tan sólo un 16.6\% de la población de 1969 estuvo formada por inmigrantes adultos. La tasa espectacular de crecimiento poblacional ha estado acompañada por una transformación del aspecto físico de la comunidad, principalmente como resultado de la actividad de los misioneros y por algunas alteraciones sutiles en la organización social. Algunos rasgos deesa transformación incluyen 
un arreglo más formal de las casas a lo largo de una pista de aterrizaje que fue solicitado por los misioneros, la construcción de una escuela con tablas de madera, y el levantamiento de un asta de bandera por los misioneros. Los enlaces familiares se han debilitado y el trabajo colectivo se hace más por pago en dinero que por colaboración de afinidad. Otro acontecimiento es la aparición de casas nucleares de familias neolocales y la desaparición de los viejos modelos de residencias.

La situación actual (1969) es resultado de muchos años de bruscos cambios culturales. Los principales hechos que aceleraron el proceso son: la formación de la comunidad con el apoyo de misioneros adventistas cerca de 1930; el ingreso al ejército peruano de varios hombres de la comunidad durante la década de los 50, dio como resultado que muchos aprendieran castellano elemental; y el desarrollo del Hospital Amazónico a principio de la década de 1960 acompañado por atención médica intensa y empleo a indígenas por parte del Hospital. El Hospital también listó a las autoridades de la comunidad que aceptaban un rebaño de ganado y la consiguiente tala del bosque para la creación de terreno de pastores. Se probó que ni el ganado ni el pasto eran productivos. Finalmente el ganado fue llevado por la creciente.

\section{MATERIALES Y METODOS}

En 1969, realicé un censo cuidadoso, al mismo tiempo una encuesta general de salud de la comunidad, con entrevistas casa por casa. Se entrevistó en castellano al hombre más viejo y todavía activo, 0 a otro lider reconocido de cada familia. Alguna información se consiguió directamente en Shipibo y cuando fue necesario, recurrí a la traducción. Recogimos datos demográficos y socioeconómicos. Se determinó la edad de cada persona con la precisión que fue posible por medio de documentación, cuando la hubo, o por conversación con la familia, cuando no la hubo. Algunas veces, se dispuso de partidas de bautismo o nacimiento o documentos del servicio militar. Las edades, declaradas por personas de 45 años o mayores, parecían no recordarse bien, por lo tanto fueron consideradas como datos inciertos.

Las fechas en que ocurrieron los hechos vitales más importantes del año anterior, fueron relacionados con las Fiestas Patrias (28 de julio de 1968). Ambos censos, 1964 y 1969, se realizaron exactamente en la misma fecha.

En 1964 y 1969 se obtuvo una historia reproductiva detallada de cada mujer, a cada una se le interrogó sobre el uso de anticonceptivos de origen vegetal o sintético. 
En 1964 no habían sido censados 115 residentes; pero sí lo fueron en 1969. Los habitantes del pueblo presentes y censados, están identificados como "cohorte censada en 1964". Losidentificados en 1969, que habían estado presentes en 1964, pero que no fueron censados en 1964, se les identificó como "cohorte no censada en 1964". La fecundidad de ambas cohortes de 1964, separada y combinada, está descrita y comparada con la población del censo de 1969, en conjunto. Los resultados del censo de 1964, están resumidos en la Tabla 4.

\section{RESULTADOS}

El censo realizado durante el mes de julio de 1969 reveló una población permanente de 549 habitantes viviendo en Paococha o Yarinacocha. La pirámide de edades y sexos (Fig. 1) indica que la población es muy joven.

La edad promedio de la población en el censo de 1969 fue de 18.3 años; la edad mediana estaba en 12.7 años. El $53.6 \%$ de la población, estaba constituida por menores de 15 años y el $61.7 \%$ tenía menos de 20 años.

Este resultado es casi idéntico al obtenido por los investigadores de la Universidad Johns Hopkins en San Antonio, Loreto, Perú, en cuanto a la distribución por edades en una comunidad de mestizos, cerca de Iquitos (Buck et al, 1968). En San Antonio, el 54.7\% de la población tenía menos de 15 años de edad, y el $61.9 \%$ menos de 20 años de edad. Hay un alto grado de similitud demográfica entre la comunidad mestiza de San Antonio, en la que el 97.7\% de los habitantes reconoce que el castellano es su idioma nativo, y la comunidad Shipibo de Paococha, en la que solamente el $3.5 \%$ de los habitantes indica que el castellano es su idioma nativo.

La edad promedio de los padres de la familia nuclear, fue alta ( 34.7 años), si se considera la juventud de la población. La edad mediana de los padres de familia, fue de 33.2 años. Un total de 102 familias nucleares, estaban organizadas en 69 unidades habitacionales y 33 complejos familiares ampliados. Los complejos familiares ampliados fueron esencialmente las mismas 37 agrupaciones de cocina.

El número promedio de personas por casa (7.9) era casi idéntico al número mediano por casa (7.7), demostrando una uniformidad notable en el tamaño de las casas familiares. Las edades promedio y mediana de los padres de las casas familiares (38.8 años) eran un poco más altas que las edades de 
padres de familias nucleares (37.1 años), consecuencia del hecho que las parejas jóvenes frecuentemente quedaban en la casa de la desposada después de casarse.

Cálculos hechos de la composición de las casas familiares señalan la fuerza de la familia extendida. Un $80 \%$ de las casas familiares contuvieron tres o cuatro generaciones, y un $85 \%$ de los individuos vivían en casas familiares que contenían las tres o cuatro generaciones. Al rehacer los cálculos de referencia a complejos familiares en lugar de casas familiares, los dos resultados cambiaron en casi $100 \%$.

Predominó el modelo matrilocal y neolocal de residencia ( $60.9 \%$ y $27.5 \%$, respectivamente), aunque en $10.1 \%$ de las casas fueron patrilocales. La monogamia era la estructura familiar más común para muchas $(87 \%)$ de las casas familiares, con la poliginia representada sólo en un $7.1 \%$ del total de casas familiares. Las casas familiares políginas, en promedio, tenían más individuos; casi un $11 \%$ de los individuos vivían en casas con estructura polígina.

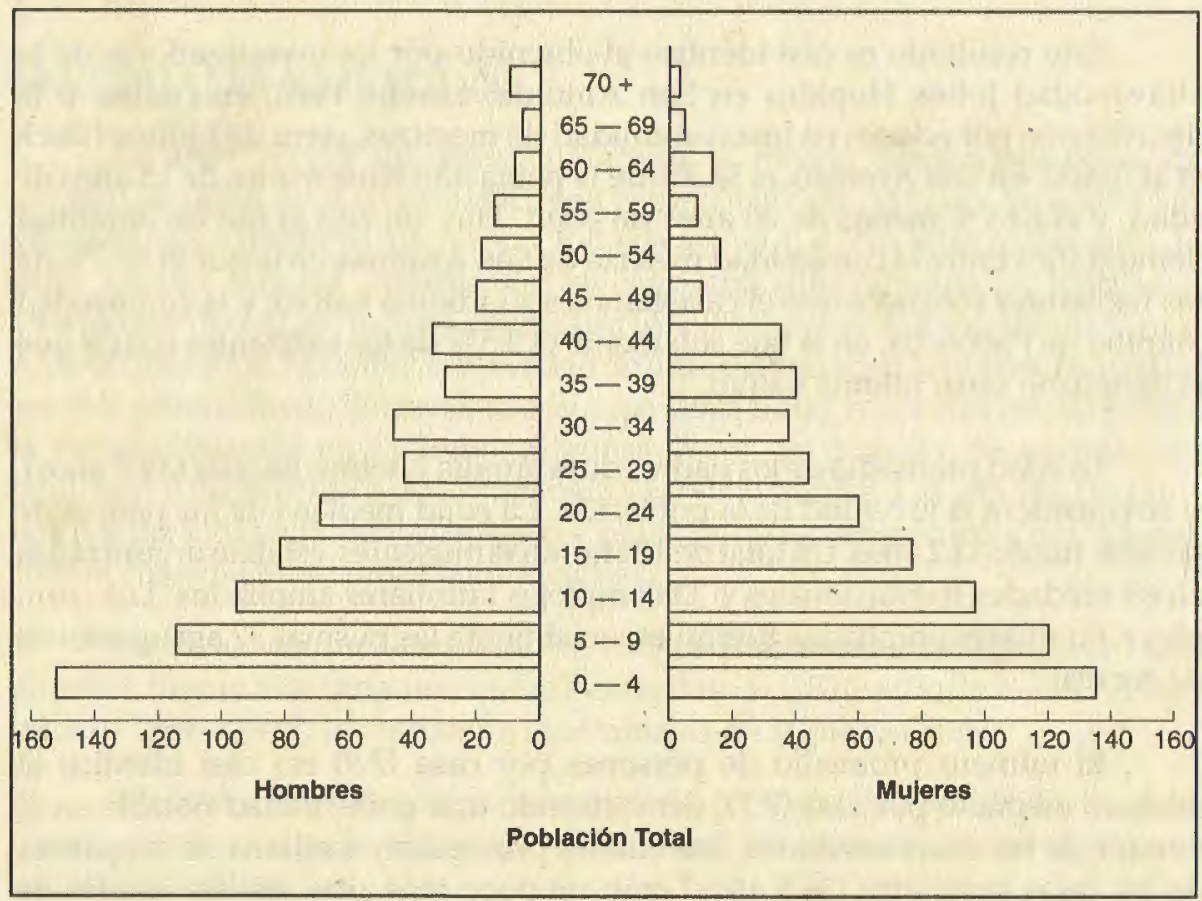


El matrimonio sororal era más común que la poliginia y tenía un nivel bajo de asociación con la poliginia. La cohabitación ocurrió a edad temprana, especialmente para las mujeres: el $89.4 \%$ de las mujeres que tenían más de 12 años ya se habían casado, y el $96.9 \%$ de las mayores de 15 años, ya eran casadas. Además, solamente el $84.6 \%$ de las mujeres ya-casadas con más de 15 años estaban actualmente cohabitando. El $9.8 \%$ de todas las mujeres casadas, estuvieron dentro de una cohabitación de poliginia corriente. Las edades media y mediana declaradas para el primer casamiento de las mujeres fue de 14.6 y 14.2 años de edad, respectivamente.

La edad media del primer parto fue 15.9 años y la modal informada fue de 15 años. Un $96.3 \%$ de las mujeres que tenían 15 años o más, informaron que habían estado embarazadas al menos una vez. Las mujeres que tenían 10 años o más, tenían un promedio de 3.1 descendientes vivos cada una, y todas las que tenían 15 años o más, el promedio fue de 3.3 descendientes vivos. El porcentaje de niños vivos, del total de nacidos, se calculó por cada mujer adulta, y el promedio fue de $68.25 \%$.

Las mujeres que tenían 15 años o más, experimentaron un promedio de 5.5 embarazos ( 5.0 partos y 0.5 abortos). Cincuentinueve embarazos terminaron en muerte fetal y están incluídos en la clasificación como abortos, con el resultado que la tasa de mortalidad fetal fue de $8.5 \%$ entre los embarazos que no llegaron a término en la población de mujeres censadas en 1969. (La definición de muerte fetal usada aquí incluye tanto los abortos inducidos y espontáneos como también los que nacieron muertos.) La fecundidad promedio completa de todas las mujeres de 45 años y mayores fue 7.5 .

Información confiable con respecto a la duración de la abstinencia sexual y la lactancia fue difícil conseguir. Los reportes varían desde inmediatamente después del parto hasta una abstinencia de 6 meses. Información sobre la duración de la lactancia variaba entre 9 meses y 3 años.

La esterilidad es insólita; dos mujeres contaron que son estériles y solamente cinco mujeres pueden considerarse subfecundas en comparación a sus padres.

\section{INDICES VITALES Y FECUNDIDAD}

Cuarentiún nacimientos y 14 muertos fueron registrados en Paococha, durante el año del censo (de 01 de Agosto de 1968 al 31 de julio de 1969). Siete 
de los fallecidos fueron niños que tenían 1-14 años de edad, y 4 eran niños con menos de un año (dos muertes neonatales).

Basado en la población censada el 1 de Agosto de 1969, la población media del año (al 1 de febrero de 1969), fue calculada en 538.5. Usando 539 como población media del año, la tasa bruta de mortalidad fue de 26.0.por 1,000 y la tasa bruta de nacimientos fue de 76.1 por 1,000 , dando por resultado una tasa bruta de incremento natural de $5.01 \%$ por año.

El tiempo calculado para que una población doble con esta tasa de incremento poblacional es de 13.8 años.

La mortalidad infantil fue de 97.5 por 1,000 nacidos vivos $(4 / 41)$, con una tasa de mortalidad neonatal de 48.8 por 1,000 . La tasa de mortalidad juvenil (14 años) fue de 75 por 10,000 y la tasa de mortalidad perinatal fue de 11.4 por 1,000 eventos perinatales (muertes fetales tardías + fallecidos durante la primera semana de nacimiento). La tasa de mortalidad fetal (muertes fetales por 1,000 habitantes) fue de 5.5 y la proporción de muertes fetales (relación de muertes fetales/nacidos vivos) fue de 78.2 por 1,000. La tasa proporcional de mortalidad (número de muertes de personas con 50 años o más en relación con el número total de muertes) fue de 7.1 por 100.

De un total de 127 mujeres con 15 años o más en la población total de 552 al 01 deagosto de 1969, 111 tenían en tre 15 y 49 años de edad. De las 111 mujeres en edad reproductiva, $13.5 \%(\mathrm{~N}=15)$ reveló presencia de embarazo actual durante la entrevista. Estas 111 mujeres han tenido 41 partos vivos, dando una tasa de fecundidad general de 0.369 . La proporción efectiva de fecundidad también fue alta (1.09); ambos índices son casi idénticos con los registrados en 1964. La tasa de fecundidad global, calculada de la suma de tasas de natalidad específica por edad, fue de 10.46 y la tasa bruta de reproducción, basada en la aplicación de la proporción de nacimientos femeninos a todas las tasas de nacimientos específicos por edad, fue de 4.974 (Tabla I).

Para tratar a las mujeres del censo de 1969 como una cohorte de 5 años, se contó el número total de nacidos vivos a las 111 mujeres en la agrupación de edad 15-49. De las 111 mujeres, 108, ó 97.3\%, han dado a luz desde 1964, en un total de 156 nacidos vivos, o 31.2 nacimientos por año. La tasa promedio de 5 años de la tasa general de fecundidad (número de nacidos vivos / número de mujeres de edades 15-49) se calculó en 31.2/111-.284 para el grupo entero. Este cálculo supone fecundidad continua en todas las agrupaciones de edad de la cohorte de 1964 a 1969. 
TABLA I. Tasa de Fecundidad Global y Tasa Bruta de Reproducción para el Censo de 1968-69, Paococha

\begin{tabular}{|c|c|c|c|c|c|}
\hline Edad & $\mathrm{N}$ & $\begin{array}{c}\text { Nacimientos } \\
\text { masculinos }\end{array}$ & $\begin{array}{c}\text { Nacimientos } \\
\text { femeninos }\end{array}$ & $\begin{array}{c}\text { Tasa de } \\
\text { natalidad }\end{array}$ & $\begin{array}{l}\text { Tasa de natalidad } \\
\text { femenina }\end{array}$ \\
\hline $15-19$ & 21 & 4 & 3 & .333 & .1429 \\
\hline $20-24$ & 23 & 7 & 8 & .652 & .3478 \\
\hline $25-29$ & 20 & 7 & 2 & .450 & .1000 \\
\hline $30-34$ & 16 & 3 & 3 & .375 & .1875 \\
\hline $35-39$ & 15 & 1 & 2 & .200 & .1333 \\
\hline $40-44$ & 12 & 0 & 1 & .082 & .0833 \\
\hline \multirow[t]{2}{*}{$45-49$} & 4 & 0 & 0 & .000 & .0000 \\
\hline & 111 & 22 & 19 & $\begin{array}{r}2.092 \\
\times 5\end{array}$ & .9948 \\
\hline \multicolumn{3}{|c|}{ Tasa de Fecundidad global } & & 10.460 & $\times 5$ \\
\hline Tasé & a d & ducción & & & $=4.9740$ \\
\hline
\end{tabular}

Otro método más preciso para determinar la tendencia de fecundidad de 5 años es analizar la experiencia de las personas que han permanecido en la comunidad desde 1964. De las 254 personas enumeradas en 1964, 209 estaban enumeradas en el censo de 1969. Trece han muerto y 32 han emigrado (Tabla II). No tuve información sobre la supervivencia o reproducción de los 32 que han emigrado, incluyendo los 12 sobrevivientes de los dos difuntos. Como consecuencia de esta falta de información, 2 de las 13 muertes de la cohorte enumerada en 1964 no están incluidas en los cálculos delas tasas vitales deesta cohorte. 
Amazonia Peruana

TABLA II. Diagrama de Censo, 1964-1969

1964

254 Enumerado

-13 Fallecido desde 1964

-32 Emigraron-no hay

conocimiento de sobrevivencia

o reproducción (incluye 12

sobrevivientes de 2 que se murió)

209 Sobrevivientes

+69 Vástagos sobrevivientes

\{81 nacidos $\}$

\{-12 muertos $\}$

69
115 No enumerado

-6 Fallecido desde 1964
109 Sobrevivientes

+43 Vástagos sobrevivientes

\{53 nacidos\}

$\{-10$ muertos $\}$

43

278

152

278

$+152$

430 Habitantes permanentes enumerados en 1969 censo

+122 Inmigrantes más vástagos enumerados en 1969 censo

522 Enumerados en censo de 1969

( $549=\mathrm{N}$ para computaciones de computadora) 
Entre los 220 miembros de la cohorte enumerada en 1964 que fueron incluidos en el censo de 1969, hubo un total de 81 nacimientos desde el censo de 1964; de eillos, unos 69 vástagos sobrevivían. Excluyendo los dos muertos, cuyos sobrevivientes ya han emigrado hace tiempo, los 200 originales también han experimentado 11 muertes, en un total de 23 muertes. Contando los 209 sobrevivientes, de la cohorte enumerada en 1964, más los 69 vástagos sobrevivientes quedó una población de 278 en 1969 (Tabla II). La población promedio de la cohorte de 5 años censada en 1964 se calculó en 243.5. Utilizando esta cifra promedio de población de 5 años, la cohorte enumerada en 1964 experimentó una tasa bruta de natalidad de 66.5 por 1,000 y una tasa bruta de mortalidad de 18.9 por 1,000. La tasa bruta de incremento natural se calculó en $4.76 \%$, dando como plazo para que la población se doble en poco menos de 15 años.

El análisis de la experiencia de fecundidad de las 57 mujeres de la cohorte enumerada en 1964 que se encontraron en 1969 en el grupo de edad de 15-49 mostró una tasa de fecundidad general de 0.284 y una proporción de fecundidad efectiva de 1.21. La tasa global de fecundidad para este grupo fue 8.191 y la tasa bruta de reproducción fue 4.082 .

Ciento, quince individuos presentes en 1964 no fueron enumerados entonces, pero fueron contados en 1969. De estos, seis habían muerto para 1969, y el grupo había tenido 53 nacimientos con 43 vástagos sobrevivientes en 1969. Con una población de 115 en 1964 y una población de 152 en 1969, la cohorte no enumerada de 1964 ha tenido una población promedio durante 5 años de 133.5 . Basado en esta cifra promedio de población de 5 años, se calculó que la cohorte no-enumerada en 1964 experimentó una tasa bruta de natalidad de 79.4 por 1,000 y una tasa bruta de mortalidad de 24.0 por mil. La tasa bruta de incremento natural de este grupo desde 1964 se calculó a 5.54\%.

El análisis de experiencia de fecundidad de 5 años de la cohorte noenumerada en 1964 reveló una tasa general de fecundidad de 0.354 y una proporción de fecundidad efectiva de 1.433. La tasa global de fecundidad del grupo de 30 mujeres de edades de 15 - 49 en 1969 fue 10.134 y la tasa bruta de reproducción fue 4.833 .

Para una evaluación más precisa de la fecundidad durante 5 años de la gente que vivió en la comunidad desde 1964, los datos de ambas cohortes enumeradas y no enumeradas en 1964 fueron combinados en dos formas. El primer juego de cálculos excluye los dos difuntos, cuyos 12 sobrevivientes emigraron permanentemente de la comunidad y cuyas experiencias de sobrevivencia y reproducción son desconocidas. El segundo método incluye los dos difuntos y sus sobrevivientes y supone sobrevivencia y ausencia de reproducción. 
Utilizando el primer método de cálculo, un total de 335 individuos que vivían en Paococha en 1964. Experimentaron un total de 134 nacimientos y 39 muertes a la fecha del censo de 1969, dando una tasa bruta de natalidad de 69.3, una tasa bruta de mortalidad de 20.4 , y una tasa bruta de incremento natural de 4.89\%. La tasa general de fecundidad para las 87 mujeres en edad de 15 - 49 al momento del censo de 1969 había sido 0.305 y la proporción de fecundidad efectiva fue 1.30. Para este grupo, usando el número promedio de mujeres en cada grupo de edad durante los 5 años, la tasa global de fecundidad fue 9.935 y la tasa bruta de reproducción fue 4.933 (Tabla III). El segundo método de cálculo, que necesariamente excluyó muchas medidas de fecundidad debido a la falta de información, reveló una tasa bruta de natalidad de 68.7, una tasa bruta de mortalidad de 21.2 , y una tasa bruta de incremento natural de $4.75 \%$ por año.

TABLA III. Tasa Global de Fecundidad y Tasa Bruta de Reproducción Combinada de 5 Años para 1964 Cohortes Enumeradas y No Enumeradas

\begin{tabular}{|c|c|c|c|c|c|}
\hline Edad & $\mathrm{N}^{\mathrm{a}}$ & $\begin{array}{l}\text { Nacimientos } \\
\text { masculinos }\end{array}$ & $\begin{array}{l}\text { Nacimientos } \\
\text { femeninos }\end{array}$ & $\begin{array}{c}\text { Tasa de } \\
\text { natalidad }\end{array}$ & $\begin{array}{l}\text { Tasa de natalidad } \\
\text { femenina }\end{array}$ \\
\hline $15-19$ & 20 & 10 & 13 & 1.150 & .650 \\
\hline $20-24$ & 17 & 23 & 19 & 2.471 & 1.118 \\
\hline $25-29$ & 14 & 20 & 7 & 1.929 & .500 \\
\hline $30-34$ & 13 & 10 & 8 & 1.385 & .615 \\
\hline $35-39$ & 10 & 7 & 13 & 2.000 & 1.300 \\
\hline $40-44$ & 4 & 0 & 3 & .750 & .750 \\
\hline \multirow[t]{3}{*}{$45-49$} & 4 & 1 & 0 & .250 & .000 \\
\hline & 82 & 71 & 63 & 9.935 & 4.933 \\
\hline & & $\begin{array}{l}\text { Tasa global } \\
\text { Tasa bruta }\end{array}$ & $\begin{array}{l}\text { e fecundidad } \\
\text { reproducció }\end{array}$ & $\begin{array}{l}=9.935 \\
=4.933\end{array}$ & \\
\hline
\end{tabular}

$\mathrm{N}^{\mathrm{a}}$ es el número promedio de mujeres en cada grupo de edad de 1964-1969 
TABLA IV. Tabla de Fecundidad Comparativa, Edades 15-49

\begin{tabular}{lcccccccc}
\hline & TBN & TBM & TCI & TFG & TEF & TGF & TBR & FCP \\
\hline Paococha (1964 & 184.7 & 36.3 & 48.4 & .362 & 1.03 & 10.065 & 4.530 & 9.8 \\
Paococha (1969) & 76.1 & 26.0 & 50.1 & .369 & 1.09 & 10.460 & 4.974 & 7.5 \\
Paococha (5 a.) & & & & & & & & \\
1964 enumerada & 66.5 & 18.9 & 47.6 & .284 & 1.21 & 8.191 & 4.082 & 9.3 \\
1964 no-enum. & 79.4 & 24.0 & 55.4 & .354 & 1.43 & 10.134 & 4.833 & \\
1964 combinada & 69.3 & 20.4 & 48.9 & .305 & 1.30 & 9.935 & 4.933 & 9.3 \\
S. Antonio & & 40.2 & .212 & 1.15 & & & & \\
Hutterites & 45.9 & 4.4 & 41.5 & .198 & .963 & & 4.0036 & 10.4 \\
Cocos-Keeling & 57.7 & 19.9 & 37.8 & .239 & .854 & & 4.212 & 8.4 \\
Atitecos & 52.4 & 23.0 & 29.4 & .232 & .950 & 8.2 & 4.000 & 9.2 \\
Colombia, 1964 & 49.3 & 14.0 & 35.3 & .211 & .874 & 8.8 & 4.20 & \\
Estados Unidos & & & & & & & & \\
1967 & 17.8 & 9.4 & 8.4 & .088 & & 2.573 & 1.255 & 2.9 \\
\hline
\end{tabular}

Definición de términos:

Tasa Bruta de Natalidad (TBN): $\frac{\text { número de nacidos vivos }}{\text { población medio total del año }} \times 1000$

Tasa Bruta de Mortalidad (TBM): $\frac{\text { número de defunciones }}{\text { población medio total del año }} \times 1000$

Tasa Bruta de Crecimiento Intrínseca (TCI): $\quad$ TBN - TBM

Tasa de Fertilidad General (TFG): Número de nacidos vivos número de mujeres de edades 15-49

Tasa Global de Fecundidad (TGF)

Tasa Bruta de Reproducción (TBR)

Fertilidad Completado Promedio (FCP) : número promedio de nacidos vivos de mujeres de edad $50+$ completado de fecundidad. 
Parece que el juego de cálculos más confiable para mostrar la alta fecundidad continua de la cohorte de 1964 durante los 5 años es el análisis de la cohorte combinada, que excluye los dos difuntos y sus 12 sobrevivientes. La comparación de varios resultados del análisis de los datos de Paococha se observan en la Tabla IV al lado de índices de fecundidad de otras poblaciones semejantes que han mostrado alta fecundidad. Es obvio que la población de Paococha tiene los índices más altos. de fecundidad, documentada en cada categoría.

\section{DISCUSION}

Asumiendo que ciertas' costumbres para controlar la natalidad y fecundidad fueron comunes antiguamente entre los Shipibo (Weisbard, 1957), aparentemente ya no son practicadas o no son efectivas en la actualidad. Hay muy pocas pruebas que señalen que el uso de anticonceptivos vegetales tengan eficacia (Hern, 1976), aun cuando hayan informes al contrario (Maxwell, 1970).

No hay prueba de que el uso de anticonceptivos vegetales tenga algún efecto (para más o menos) sobre la fecundidad global de las mujeres del pueblo. La reputación de eficacia de los anticonceptivos vegetales puede originarse en el uso, dentro de un ambiente polígino, junto con la abstinencia sexual prolongada, después del parto. Es decir, una relación estadística entre el uso de anticonceptivos vegetales y una paridad promedio más baja, puede ser simplemente una relación secundaria no causal.

En un estudio ya publicado, (Hern 1976) revelé una colección conocida de conceptos y creencias, mantenida por los Shipibo, que pertenecen a la Anatomía y Fisiología de la Reproducción, tanto como un fuerte deseo de controlar la fertilidad. La disminución en la eficacia aparente de los anticonceptivos vegetales, puede estar ligada con la declinación en la prevalencia de la poliginia.

La importancia de la poliginia, en la cultura Shipibo, está demostrada dramáticamente por el hecho quelas tentativas delos misioneros para disuadirlos de estas costumbres, fueron las causas principales de matanza de misioneros por algunos Shipibo, en 1697 (Steward \& Metraux, 1948). Aunque no hay documentación adecuada de la prevalencia de la poliginia en comunidades más tradicionales, puede ser dos veces más de la prevalencia en comunidades como Paococha. Abelov informó sobre una prevalencia de $40 \%$ de asociación de 
uniones políginas entre las mujeres de una comunidad Shipibo no identificada en 1977 (Abelov, 1978).

Varios habitantes nos informaron de una declinación en la prevalencia de poliginia en la comunidad. Whiting (1964) y Lorimer (1954) señalan que la poliginia es común en las sociedades preindustriales más complejas y que hay una relación entre poliginia, patrilocalidad, y tabú post-partum sexual prolongado. Dorjahn (1958) asevera que el efecto de poliginia para las mujeres es disminuir la fecundidad, pero su análisis principal está restringido a la experiencia de fecundidad de los hombres. La poliginia puede tener dos efectos: reducir el número de descendientes de cada mujer cohabitante en razón de aumentar los intervalos entre los nacimientos y aumentar la proporción de mujeres que se hayan casado o cohabitado. El primer efecto sería disminuir la fertilidad individual y comunal, y el segundo sería aumentar la fertilidad en general.

Si la poliginia resulta en fertilidad más baja, debido a intervalos más largos entre nacimientos, la declinación alegada en la prevalencia de poliginia en Paococha podría ser un factor de reducción de la duración de la abstinencia sexual post-partum y por lo tanto de los intervalos entre nacimientos. Esta puede ser la consecuencia directa del cambio cultural, porque los misioneros activamente atacaron la poliginia tanto como el infanticidio. Es difícil ver cómo la proporción de mujeres que se hayan casado o cohabiten en Paococha podría ser más alta de lo que es en la actualidad.

La edad temprana de la cohabitación no parece ser resultado de cambio cultural, porque muchas de las mujeres en los últimos años de reproducción señalaron que han estado entregadas a sus esposos antes de la pubertad. Era costumbre para ellas, que la actividad sexual empezara luego de la primera menstruación. Ciertamente los misioneros no favorecieron esta costumbre.

Parece no obstante que las fuerzas externas de cambio han jugado un papel significativo en producir una tasa repentinamente alta de crecimiento poblacional en Paococha. La intervención de atención médica occidental probablemente es el factor principal en la disminución en las tasas de mortalidad materna einfantil. Una disminución en la tasa demortalidad materna puede ser altamente significativa en una sociedad como ésta, que se caracteriza por una paridad temprana y prolongada, casamiento o cohabitación casi universal, y alta fecundidad. También es posible que los factores sociales hayan jugado un papel importante si la prevalencia de la poliginia ha disminuido $y$, junto con esto, la duración de los intervalos entre nacimientos. 
El ambiente biótico también ha cambiado, pero documentar esto en forma detallada es más difícil. Los habitantes de Paococha señalan no obstante, que la caza es significativamente más escasa que hace 10 ó 15 años, aun en el monte más lejano. La gente misma atribuye estas diferencias a la presión de aumento de población: hay más gente buscando su rancho y los animales y pescados son capturados antes de la madurez. La deforestación afecta no solamente las condiciones del suelo sino que ha reducido el área de habitación para la vida silvestre. La gente dice que es más difícil conseguir carne del monte y debido a esto tienen que comprar gallinas y cultivar arroz para pagar las cuentas. Las inundaciones cada vez más fuertes del río hacen más favorable el cultivo de cosechas de pago como el arroz.

Puede pasar mucho tiempo antes de que se conozca y entienda todos los efectos de la tasa repentina de crecimiento de población en la situación social de Paococha y su ambiente adyacente. Tal vez no sería posible entender los efectos sin considerar el contexto en el cual ocurre: específicamente, la alta fecundidad y la rápida destrucción del ambiente en comunidades cercanas o aún lejanas, algunas indígenas y algunas no indígenas. Por ahora, solamente hay una vaga sensación de quebrantamiento social e interrupción de la tranquilidad de la comunidad. Hay una percepción clara de que los pescados son más chicos y más difíciles de pescar que hace pocos años, y que es más difícil conocer a todos y mantener relaciones amistosas con los vecinos.

* Este artículo ha sido publicado en una primera versión en inglés bajo el mismo título en la revista Human Ecology, Vol. 5, № 4, 1977. Esta versión en castellano está a cargo del mismo autor. 


\section{BIBLIOGRAFIA}

AGUALIMPIA, C,M.; PABON, A.R.; GALAN, R.M.; GOMEZ S., L.C., y GONZALEZ, L.E.

1969. Demographic facts of Colombia. Milbank Memorial Fund Quarterly 47:255-296.

BUCK, A.A.; SASAKI, T.T, y ANDERSON, R.I.

1968 Health and Disease in Four Peruvian Villages. Johns Hopkins Press, Baltimore.

COLLVER, O.A.

1965. Birth Rates in Latin America.University of California, Berkeley.

DORJAHN, V.R.

1958. Ferfility, polygyny, and their interrelations in Temne society. American Anthropologist $\quad 60: 838-860$.

EARLY, J.D.

1970. Demographic profile of a Maya community. Milbank Memorial fund Quarterly 48(2):167-178.

EATON, J.W., MAYER, A.J.

1953. The social biology of very high fertility among the Hutterites. Human Biology 25:206-264.

HERN, W.M.

1976. Knowledge and use of herbal contraceptives in a Peruvian Amazon village. Human Organization 35:9-19.

LATHRAP, D.W.

1968 The "hunting" economies of the tropical forest zone of South America: An attempt at historical perspective. In Lee, R.B., and Devore, I. (eds.), Man the Hunter. Aldine, Chicago, pp. 23-29.

LATHRAP, D.W.

1970. The Upper Amazon. Praeger, New York.

LORIMER, F.

1954. Culture and Human Fertility. UNESCO, París, pp. 58-105; 199-206. 
MAXWELL, $N$.

1970. Attitudes of four Peruvian jungle tribes towards plants employed as oral contraceptives. Presented at the 39th International Congress of Americanists, Lima, Perú, August 4, 1970 (mimeo).

NORTMAN, D.

1969. Population and Family Planning Programs: A Factbook, Reports on Population/Family Planning, no. 2. Population Council, New York.

SMITH, T.E.

1960 The Cocos-Keeling Islands: A demographic laboratory. Population Studies 14:94-130.

VITAL STATISTICS OF THE UNITED STATES, 1967

1969 Vol. I - Natality, Section 1, Tables 1-1, 1-2, 1-4, p. 13; Table 1-15, p. 115; vol. II -Mortality, Part A, Section 1, Table 1-1, p. 1-2. U.S. Department of Health, Education and Welfare, Washington, D.C.

WEISBARD, S.R.

1957 Indios Shamas de Peru. Ethnographie 53:19- 74.

WHITING, J.W.M.

1964 Effects of climate on certain cultural practices. In Goodenough, W.M. (ed.), Explorations in Current Anthropology. McGraw-Hill, New York. 